\title{
Altered fovea in AQP4-IgG-seropositive neuromyelitis optica spectrum disorders
}

Seyedamirhosein Motamedi, MSc, Frederike C. Oertel, MD, Sunil K. Yadav, PhD, Ella M. Kadas, PhD, Margit Weise, MSc, Joachim Havla, MD, Marius Ringelstein, MD, Orhan Aktas, MD, Philipp Albrecht, MD, Klemens Ruprecht, MD, Judith Bellmann-Strobl, MD, Hanna G. Zimmermann, PhD, Friedemann Paul, MD, and Alexander U. Brandt, MD

Neurol Neuroimmunol Neuroinflamm 2020;7:e805. doi:10.1212/NXI.0000000000000805

\section{Abstract \\ Objective}

To investigate disease-specific foveal shape changes in patients with neuromyelitis optica spectrum disorders (NMOSDs) using foveal morphometry.

\section{Methods}

This cross-sectional study included macular spectral domain optical coherence tomography scans of 52 eyes from 28 patients with aquaporin-4 immunoglobulin G (AQP4-IgG)-seropositive NMOSD, 116 eyes from 60 patients with MS, and 123 eyes from 62 healthy controls (HCs), retrospectively, and an independent confirmatory cohort comprised 33/33 patients with NMOSD/MS. The fovea was characterized using 3D foveal morphometry. We included peripapillary retinal nerve fiber layer ( $\mathrm{pRNFL}$ ) thickness and combined macular ganglion cell and inner plexiform layer (GCIPL) volume to account for optic neuritis (ON)-related neuroaxonal damage.

\section{Results}

Group comparison showed significant differences compared with $\mathrm{HC}$ in the majority of foveal shape parameters in NMOSD, but not MS. Pit flat disk area, average pit flat disk diameter, inner rim volume, and major slope disk length, as selected parameters, showed differences between NMOSD and MS ( $p$ value $=0.017,0.002,0.005$, and 0.033, respectively). This effect was independent of ON. Area under the curve was between 0.7 and 0.8 (receiver operating characteristic curve) for discriminating between NMOSD and MS. Pit flat disk area and average pit flat disk diameter changes independent of $\mathrm{ON}$ were confirmed in an independent cohort.

\section{Conclusions}

Foveal morphometry reveals a wider and flatter fovea in NMOSD in comparison to MS and HC. Comparison to MS and accounting for ON suggest this effect to be at least in part independent of ON. This supports a primary retinopathy in AQP4-IgG-seropositive NMOSD.
Correspondence

Dr. Brandt

alexander.brandt@charite.de 


\section{Glossary}

AQP4-IgG = aquaporin-4 immunoglobulin G; ART = automatic real time; AUC = area under the curve; $\mathbf{B}$ = estimate; FT = foveal thickness; GCIPL = combined macular ganglion cell and inner plexiform layer; HC = healthy control; ILM = inner limiting membrane; INL = inner nuclear layer; $\mathbf{M O G}=$ myelin oligodendrocyte glycoprotein; NMOSD = neuromyelitis optica spectrum disorder; OCT = optical coherence tomography; $\mathbf{O N}=$ optic neuritis; $\mathbf{O N}-=$ eyes without a history of ON; $\mathbf{O N}+=$ eyes with a history of $\mathrm{ON} ; \mathbf{p R N F L}=$ peripapillary retinal nerve fiber layer; $\mathbf{R O C}=$ receiver operating characteristic; $\mathrm{SE}=$ standard error of $\mathrm{B}$.

Aquaporin-4 immunoglobulin G (AQP4-IgG)-seropositive neuromyelitis optica spectrum disorder (NMOSD) is an inflammatory astrocytopathy defined by pathogenic serum immunoglobulin $\mathrm{G}$ antibodies against aquaporin- $4 .{ }^{1-3}$

Optic neuritis (ON) is a hallmark of NMOSD and leads to severe neuroaxonal damage in optic nerve and retina associated with oftentimes severe vision loss. ${ }^{4-8}$ Retinal optical coherence tomography (OCT) can be used to measure this damage $^{9-12}$ : Peripapillary retinal nerve fiber layer ( $\mathrm{pRNFL}$ ) and combined macular ganglion cell and inner plexiform layer (GCIPL) typically become thinner, whereas inner nuclear layer (INL) becomes thicker as a result of $\mathrm{ON}^{6,13-15}$

Recently, a foveal thickness (FT) reduction has been reported in eyes never experiencing an ON in patients with AQP4IgG-seropositive NMOSD, ${ }^{16,17}$ suggesting either subclinical optic nerve inflammation or primary retinal astrocytopathy in NMOSD. ${ }^{8}$ This change in FT appeared to be driven by a change in foveal shape, with a normally V-shaped fovea appearing more widened and $\mathrm{U}$-shaped with flattened disk in eyes of patients with AQP4-IgG-seropositive NMOSD. ${ }^{17}$

Because FT is a weak measure for foveal shape, we developed a 3D foveal morphometry method, which we previously described and validated in detail. ${ }^{18}$ Here, we use this approach to investigate the foveal shape in patients with AQP4IgG-seropositive NMOSD. We compare findings against measurements in patients with MS, which also presents with $\mathrm{ON}$, and against healthy controls (HCs). Our goal was to investigate whether foveal changes are characteristic to AQP4IgG-seropositive NMOSD and not simply caused by ON.

\section{Methods}

\section{Study population}

In this analysis, we retrospectively included data from an ongoing observational cohort study in patients with NMOSD at the NeuroCure Clinical Research Center at CharitéUniversitätsmedizin Berlin, Germany, acquired from August 2013 to November 2016. Inclusion criteria were a minimum age of 18 years and fulfilling the diagnostic criteria for AQP4IgG-seropositive NMOSD according to the 2015 International Consensus Diagnostic Criteria. ${ }^{7}$ AQP4-IgG-seropositivity was tested using a cell-based assay (Euroimmun, Lübeck, Germany). Exclusion criteria were any other neurologic or ophthalmologic disorder (e.g., glaucoma, diabetes, and refractive error $>6$ diopters), which can affect the retina. ${ }^{19}$ Eyes with an episode of $\mathrm{ON}$ within the last 6 months before the OCT examinations were excluded. Of 46 patients enclosed in the study, we included 28 patients with NMOSD in the analysis after applying the inclusion and exclusion criteria (table 1). We additionally included 60 patients with relapsing-remitting MS according to the 2010 revised McDonald criteria, ${ }^{20}$ from 2 cohort studies about MS and clinically isolated syndrome and $62 \mathrm{HCs}$, both groups age and sex matched to the NMOSD cohort, in this study (table 1). Data from 17 patients with AQP4-IgG-seropositive NMOSD $(61 \%)$ were already included in a previous study by Oertel et al. ${ }^{17}$ High-contrast visual acuity was measured using Early Treatment in Diabetes Retinopathy Study charts at a 4-m distance with an Optec 6500 P system (Stereo Optical, Chicago, IL), with best correction and under photopic conditions.

A confirmatory cohort consisting of macular OCTs from 58 eyes of 33 patients with AQP4-IgG-seropositive NMOSD (eyes with a history of $\mathrm{ON}[\mathrm{ON}+]$ : $27 ; 33$ women; age: $49.2 \pm$ 15.4 years) and 62 eyes of 33 patients with MS (ON+: 12; 32 women; age: $49.7 \pm 14.7$ years) from longitudinal prospective observational cohort studies at the Department of Neurology, Universitätsklinikum Düsseldorf at Heinrich Heine University, Düsseldorf, Germany, was included in this study, following the same inclusion and exclusion criteria. MS and NMOSD groups were well matched in this cohort for age $(p=0.812)$ and sex $(p$ $=1)$, but not for the proportion of eyes with ON $(p=0.001)$. The NMOSD group is well matched to the Berlin cohort for age $(p=0.113)$, sex $(p=0.214)$, and $\mathrm{ON}+(p=0.507)$.

\section{Ethics statement}

The study was approved by the local ethics committee at Charité-Universitätsmedizin Berlin (EA1/131/09, EA1/163/ 12 , and EA1/182/10). The confirmatory OCT data were collected under approval from the local ethics committee at Heinrich Heine University Düsseldorf (4389R). The study was conducted according to the Declaration of Helsinki in its currently applicable version and the applicable German and European laws. All the participants gave written informed consent.

\section{Optical coherence tomography}

All retinal OCT images (exploratory and confirmatory cohort) were taken using Spectralis spectral domain OCT devices from Heidelberg Engineering (Heidelberg, Germany), with activated eye tracker and automatic real-time (ART) averaging. The pRNFL thickness was calculated using standard ring scans around the optic nerve head $\left(12^{\circ}\right.$, single $B$ 
Table 1 Demographic description of NMOSD, MS, and HC cohorts

\begin{tabular}{|c|c|c|c|}
\hline & NMOSD & MS & HC \\
\hline No. of patients $(\mathrm{N})$ & 28 & 60 & 62 \\
\hline No. of eyes $(\mathrm{N})$ & 52 & 116 & 123 \\
\hline Sex (female) $(N[\%])^{a}$ & $26(93 \%)$ & $55(92 \%)$ & $56(90 \%)$ \\
\hline Age $(y)(\text { mean } \pm S D)^{b}$ & $43.6 \pm 11.5$ & $39.0 \pm 10.9$ & $41.7 \pm 13.5$ \\
\hline Patients with a history of ON (N [\%]) & $16(57 \%)$ & $29(48 \%)$ & - \\
\hline Eyes with a history of ON $(\mathrm{N}[\%])^{c}$ & $20(38 \%)$ & $32(28 \%)$ & - \\
\hline Number of ONs per eye (median [range]) & $1.5(1-8)$ & $1(1-2)$ & - \\
\hline VA for ON eyes $(\log M A R)(\text { mean } \pm S D)^{d}$ & $0.36 \pm 0.69$ & $-0.09 \pm 0.10$ & \\
\hline EDSS score (median [range]) & $3(0-6.5)$ & $2(0-4.5)$ & - \\
\hline Disease duration $(y)($ mean $\pm S D)$ & $6.8 \pm 4.8$ & $7.9 \pm 8.4$ & - \\
\hline pRNFL $(\mu \mathrm{m})($ mean $\pm S D)$ & $81.2 \pm 22.1$ & $90.8 \pm 15.3$ & $97.6 \pm 8.8$ \\
\hline GCIPL $\left(\mathrm{mm}^{3}\right)($ mean $\pm \mathrm{SD})$ & $1.69 \pm 0.29$ & $1.83 \pm 0.24$ & $1.94 \pm 0.14$ \\
\hline $\mathrm{INL}\left(\mathrm{mm}^{3}\right)($ mean $\pm \mathrm{SD})$ & $0.94 \pm 0.09$ & $0.96 \pm 0.06$ & $0.95 \pm 0.06$ \\
\hline FT $(\mu \mathrm{m})($ mean \pm SD $)$ & $262.9 \pm 14.9$ & $272.1 \pm 20.3$ & $272.3 \pm 23.1$ \\
\hline \multicolumn{4}{|c|}{ 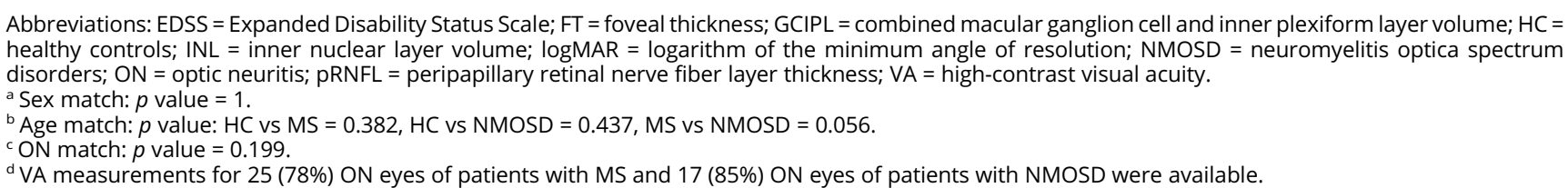 } \\
\hline
\end{tabular}

scan with 1536 A scans, $16 \leq \mathrm{ART} \leq 100)$. The volume of the GCIPL and INL was calculated in a 6-mm diameter around the fovea, and FT was measured in a 1-mm-diameter area around the fovea, based on macular volume scans $\left(25^{\circ} \times 30^{\circ}\right.$, $61 \mathrm{~B}$ scans with 768 A scans per each B scan, ART $=15$ ). Intraretinal layer segmentation was performed and corrected if needed using Heidelberg Eye Explorer (HEYEX version 1.9.10.0) by an experienced grader. All OCT scans were quality controlled according to the OSCAR-IB criteria, ${ }^{19,21}$ and OCT data are reported in accordance with the Advised Protocol for OCT Study Terminology and Elements (APOSTEL) recommendations. ${ }^{22}$ Four eyes from the exploratory cohort were excluded from the study because of inadequate OCT scan quality.

\section{Foveal morphometry}

All macular volume OCTs were analyzed using a 3D foveal morphometry described previously in detail. ${ }^{18}$ In brief, first, the 3D macular scan is flattened based on the segmentation of the Bruch membrane as the reference plane, and then the inner limiting membrane (ILM) surface is smoothed and reconstructed radially using a cubic Bezier polynomial model. Based on the reconstructed ILM surface, several parameters are defined to describe the foveal and parafoveal shape. Three surfaces are defined in this foveal shape analysis method: rim disk, which connects the points on the surface with the maximum height (rim points); slope disk, which connects the points with the maximum slopes in the parafoveal area; and pit flat disk, which characterizes the flatness of the foveal pit. Each surface is described by 4 parameters - the length in the dominant direction (major axis): major length; the length in the second dominant direction (perpendicular to the major axis): minor length; the area; and the average diameter. In addition, the distance between the fovea (the minimum point) and the center of rim disk: average pit depth; the distance between the fovea and the reference plane: central foveal thickness; the average height of the rim points: average rim height; the volume between the ILM surface and the reference plane: rim volume; the volume between the ILM surface and rim disk: pit volume; the volume between the ILM surface and the reference plane within 1-mm-diameter cylinder centered at the fovea: inner rim volume; and the average slope at the maximum slope points: average maximum pit slope are measured by this method to characterize the $3 \mathrm{D}$ foveal shape. Figure 1 gives an overview of the method and the defined parameters. Test-retest reliability was excellent in all foveal morphometry parameters with intraclass correlation coefficients $>0.9$ in all cases (table e-1, links.lww.com/NXI/A270).

\section{Statistical analysis}

Sex and ON differences between groups were tested using $\chi^{2}$ tests, and age differences were tested using 2 -sample Wilcoxon tests. Performance measurements were based on the area under the curve (AUC) for receiver operating 

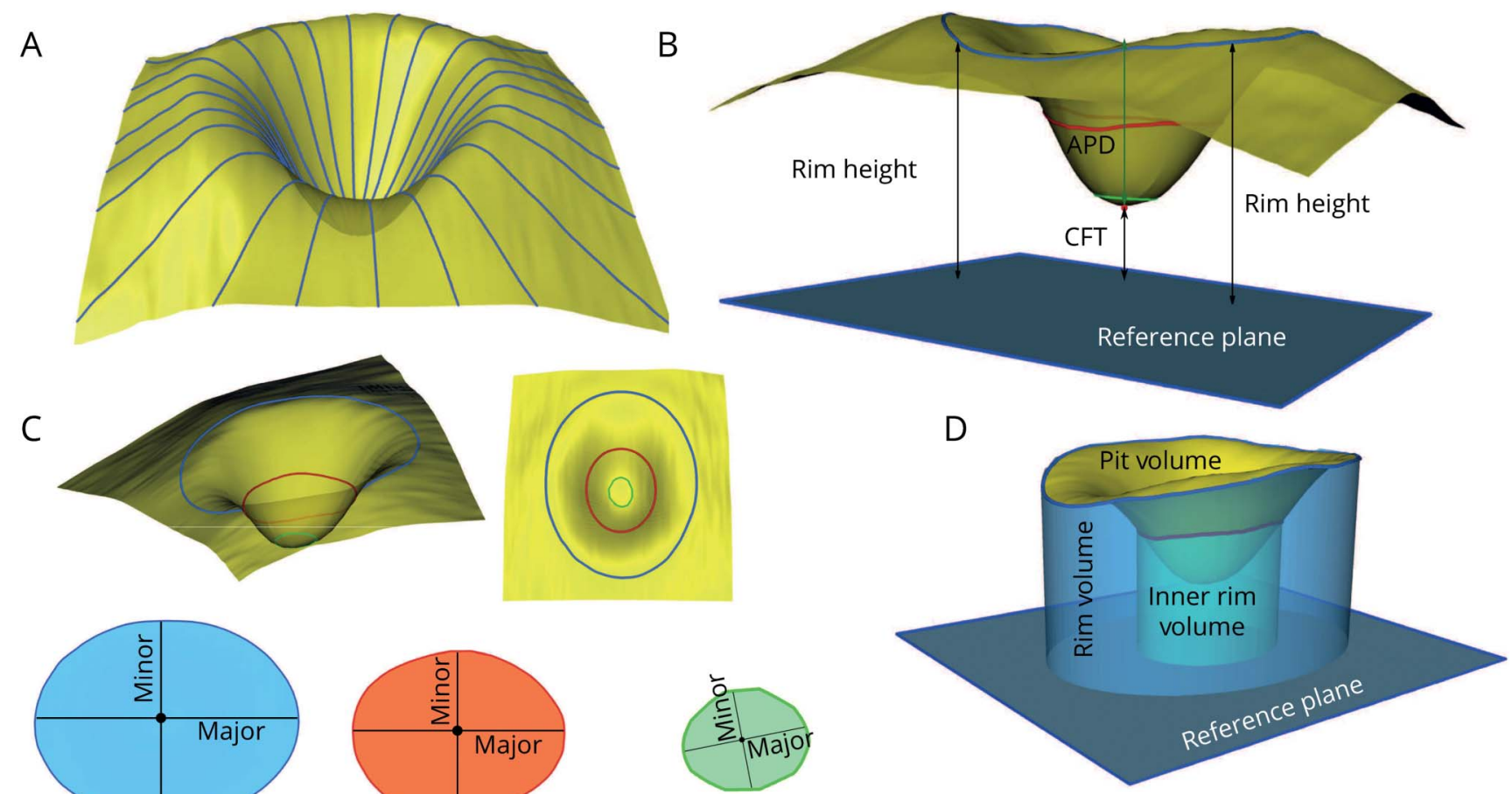

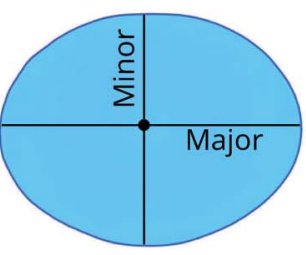

Rim disk

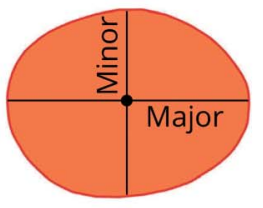

Slope disk

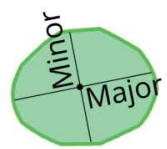

Pit flat disk

(A) ILM surface smoothing and radial reconstruction using the cubic Bezier polynomial. (B) Rim height, average pit depth, and central foveal thickness. (C) Rim disk (blue), slope disk (red), and pit flat disk (green) and major and minor axes on each surface. (D) Rim volume, pit volume, and inner rim volume. APD = average pit depth, CFT = central foveal thickness; ILM = inner limiting membrane; major = major axis; minor = minor axis.

characteristic (ROC) curves. All linear regression analyses were performed using linear mixed-effect models including intereye within-patient correlations, age, and sex as random effects. The marginal and conditional coefficients of determination of the linear models were calculated with pseudo R-squared. Stepwise logistic regression analysis for model selection was performed by the Akaike Information Criteria with both backward and forward modes of stepwise search based on generalized linear models. In this exploratory study, we corrected $p$ values for multiple testing using the BenjaminiHochberg procedure. In addition, the identified parameter differences were tested in a second independent cohort obtained with the same scanning protocol at a different center. One-sided $p$ values were reported for the confirmatory cohort, not corrected for multiple testing. Sample size for the confirmatory cohort was calculated for 1-sided 2-sample $t$-test with $90 \%$ power and a significance level of 0.05 . To adjust this estimate for eye-based statistics, we added $60 \%$ sample size to account for intereye within-patient effects and additional covariates. All statistical analysis were performed in $\mathrm{R}$ version 3.5.0 ${ }^{23}$ with packages stats, lme4, lmerTest, MuMIn, ROCR, ggplot2, plotROC, pwr, multcomp, and ggpubr packages. The $p$ values less than 0.05 were considered significant.

\section{Data availability}

All data are available on reasonable request from the corresponding author.

\section{Results}

\section{Foveal shape changes in NMOSD and MS}

First, we analyzed foveal shape in patients with NMOSD and MS and compared results with measurements in HCs. Foveal shape was altered in patients with NMOSD, but only mildly affected in patients with MS, both in comparison to HCs (table 2). Foveal parameters stratified by history of $\mathrm{ON}$ are included in supplemental data (table e-2, links.lww.com/NXI/A271).

In contrast, both patients with MS and NMOSD showed neuroaxonal damage typically occurring after ON: pRNFL and GCIPL were lower in patients with NMOSD in comparison to HCs (pRNFL: standard error of $B[S E]=-17.7[3.0] \mu \mathrm{m}$, $p<0.001$, GCIPL: $\left.\mathrm{B}[\mathrm{SE}]=-0.27[0.04] \mathrm{mm}^{3}, p<0.001\right)$, but also in patients with MS in comparison to HCs (pRNFL: B $[\mathrm{SE}]=-7.0(2.0) \mu \mathrm{m}, p<0.001, \mathrm{GCIPL}: \mathrm{B}[\mathrm{SE}]=-0.12[0.03]$ $\left.\mathrm{mm}^{3}, p=0.001\right)$.

\section{Parameter selection}

Next, we selected parameters with the highest potential to be abnormal in NMOSD: We therefore analyzed parameter performance in discriminating between eyes from patients with NMOSD and MS, regardless of ON status (table 3 and figure $2 \mathrm{~A}$ ). This was followed by a linear regression analysis against diagnosis, history of $\mathrm{ON}$, and their interaction effect to derive effect sizes and group differences accounting for ON. 
Table 2 Foveal shape analysis parameters results (mean \pm SD) and linear regression analysis for NMOSD and MS vs HC

\begin{tabular}{|c|c|c|c|c|c|c|c|}
\hline & \multirow{2}{*}{$\begin{array}{l}\text { HC (mean } \pm \\
\text { SD) }\end{array}$} & \multirow{2}{*}{$\begin{array}{l}\text { MS (mean } \pm \\
\text { SD) }\end{array}$} & \multirow{2}{*}{$\begin{array}{l}\text { NMOSD (mean } \pm \\
\text { SD) }\end{array}$} & \multicolumn{2}{|l|}{ HC vs NMOSD } & \multicolumn{2}{|l|}{ HC vs MS } \\
\hline & & & & B (SE) & $p$ & B (SE) & $p$ \\
\hline Average pit depth (mm) & $0.117 \pm 0.021$ & $0.111 \pm 0.018$ & $0.101 \pm 0.026$ & $\begin{array}{l}-0.017 \\
(0.005)\end{array}$ & $<0.001$ & $-0.006(0.003)$ & 0.078 \\
\hline Central foveal thickness (mm) & $0.231 \pm 0.015$ & $0.229 \pm 0.017$ & $0.228 \pm 0.017$ & $\begin{array}{l}-0.004 \\
(0.004)\end{array}$ & 0.330 & $-0.002(0.003)$ & 0.535 \\
\hline Average rim height (mm) & $0.348 \pm 0.014$ & $0.340 \pm 0.016$ & $0.328 \pm 0.018$ & $\begin{array}{l}-0.021 \\
(0.003)\end{array}$ & $<0.001$ & $-0.009(0.002)$ & $<0.001$ \\
\hline Average rim disk diameter (mm) & $2.184 \pm 0.115$ & $2.152 \pm 0.110$ & $2.132 \pm 0.130$ & $\begin{array}{l}-0.056 \\
(0.027)\end{array}$ & 0.037 & $-0.034(0.020)$ & 0.082 \\
\hline Rim disk area $\left(\mathrm{mm}^{2}\right)$ & $3.717 \pm 0.387$ & $3.606 \pm 0.371$ & $3.545 \pm 0.436$ & $\begin{array}{l}-0.184 \\
(0.090)\end{array}$ & 0.041 & $-0.116(0.066)$ & 0.079 \\
\hline Major rim disk length (mm) & $0.630 \pm 0.066$ & $0.613 \pm 0.063$ & $0.600 \pm 0.075$ & $\begin{array}{l}-0.032 \\
(0.015)\end{array}$ & 0.039 & $-0.018(0.011)$ & 0.112 \\
\hline Minor rim disk length (mm) & $0.619 \pm 0.065$ & $0.599 \pm 0.062$ & $0.590 \pm 0.072$ & $\begin{array}{l}-0.030 \\
(0.015)\end{array}$ & 0.042 & $-0.021(0.011)$ & 0.055 \\
\hline Average slope disk diameter $(\mathrm{mm})$ & $0.663 \pm 0.119$ & $0.653 \pm 0.152$ & $0.771 \pm 0.136$ & $0.104(0.028)$ & $<0.001$ & $-0.012(0.025)$ & 0.626 \\
\hline Slope disk area $\left(\mathrm{mm}^{2}\right)$ & $0.361 \pm 0.131$ & $0.358 \pm 0.180$ & $0.486 \pm 0.164$ & $0.120(0.032)$ & $<0.001$ & $-0.005(0.028)$ & 0.858 \\
\hline Major slope disk length (mm) & $0.068 \pm 0.025$ & $0.068 \pm 0.034$ & $0.090 \pm 0.029$ & $0.021(0.006)$ & $<0.001$ & $-4.8 e^{-4}(0.005)$ & 0.930 \\
\hline Minor slope disk length (mm) & $0.053 \pm 0.019$ & $0.052 \pm 0.026$ & $0.072 \pm 0.026$ & $0.019(0.005)$ & $<0.001$ & $-0.001(0.004)$ & 0.772 \\
\hline $\begin{array}{l}\text { Average pit flat disk diameter } \\
(\mathrm{mm})\end{array}$ & $0.215 \pm 0.030$ & $0.211 \pm 0.039$ & $0.257 \pm 0.052$ & $0.042(0.008)$ & $<0.001$ & $-0.005(0.006)$ & 0.440 \\
\hline Pit flat disk area $\left(\mathrm{mm}^{2}\right)$ & $0.037 \pm 0.010$ & $0.036 \pm 0.015$ & $0.054 \pm 0.025$ & $0.017(0.003)$ & $<0.001$ & $-0.001(0.002)$ & 0.691 \\
\hline Major pit flat disk length (mm) & $\begin{array}{l}0.0067 \pm \\
0.0018\end{array}$ & $\begin{array}{l}0.0065 \pm \\
0.0027\end{array}$ & $0.0098 \pm 0.0048$ & $\begin{array}{l}0.0032 \\
(0.0007)\end{array}$ & $<0.001$ & $\begin{array}{l}-0.0001 \\
(0.0004)\end{array}$ & 0.725 \\
\hline Minor pit flat disk length (mm) & $\begin{array}{l}0.0058 \pm \\
0.0016\end{array}$ & $\begin{array}{l}0.0056 \pm \\
0.0023\end{array}$ & $0.0083 \pm 0.0036$ & $\begin{array}{l}0.0026 \\
(0.0005)\end{array}$ & $<0.001$ & $\begin{array}{l}-0.0002 \\
(0.0004)\end{array}$ & 0.628 \\
\hline Rim volume $\left(\mathrm{mm}^{3}\right)$ & $1.045 \pm 0.153$ & $0.983 \pm 0.133$ & $0.910 \pm 0.170$ & $\begin{array}{l}-0.141 \\
(0.036)\end{array}$ & $<0.001$ & $-0.061(0.025)$ & 0.013 \\
\hline Inner rim volume $\left(\mathrm{mm}^{3}\right)$ & $0.104 \pm 0.018$ & $0.103 \pm 0.019$ & $0.088 \pm 0.015$ & $\begin{array}{l}-0.017 \\
(0.004)\end{array}$ & $<0.001$ & $-0.001(0.003)$ & 0.753 \\
\hline Pit volume $\left(\mathrm{mm}^{3}\right)$ & $0.252 \pm 0.043$ & $0.246 \pm 0.051$ & $0.259 \pm 0.044$ & $0.005(0.010)$ & 0.606 & $-0.007(0.008)$ & 0.402 \\
\hline $\begin{array}{l}\text { Average maximum pit slope } \\
\text { (degrees) }\end{array}$ & $12.16 \pm 3.38$ & $11.10 \pm 2.41$ & $9.86 \pm 3.11$ & $-2.42(0.74)$ & 0.001 & $-1.03(0.52)$ & 0.047 \\
\hline
\end{tabular}

Abbreviations: $\mathrm{B}=$ estimate; $\mathrm{HC}=$ healthy controls; $\mathrm{MS}=$ patients with MS; NMOSD = patients with neuromyelitis optica spectrum disorders; $\mathrm{SE}=$ standard error of B.

Significant $p$ values are marked in bold.

Table 3 shows the results of this selection process, ordered by AUC. The best parameter selected from the ROC analysis was pit flat disk area (AUC $=0.798$, figure $2 \mathrm{~A})$. To derive a final set of relevant parameters, we computed a stepwise logistic regression model to predict NMOSD vs MS, including only the parameters with $A U C \geq 0.7$. This selected 4 parameters: pit flat disk area, average pit flat disk diameter, inner rim volume, and major slope disk length (figure 2, D-G).

\section{Association with ON and neuroaxonal damage} A crucial question is whether these parameters react to $\mathrm{ON}$ related damage or are indeed at least partially independent. To further investigate this, we repeated the AUC analysis, but this time separately for the eyes without a history of $\mathrm{ON}(\mathrm{ON}-)$ and the $\mathrm{ON}+$ (table e-2, links.lww.com/NXI/A271). Indeed, foveal shape was altered also in the ON-. Here, the best foveal shape parameter to distinguish ON- from patients with NMOSD and patients with MS was minor pit flat disk length (AUC $=0.804$, figure $2 \mathrm{~B}$ ). In $\mathrm{ON}+$, the best-performing parameter to discriminate between patients with NMOSD and patients with MS was major pit flat disk length (AUC $=0.817$, figure $2 \mathrm{C}$ ). Of note, NMOSD ON- also showed signs of mild neuroaxonal damage compared with HC (pRNFL: B [SE] $=-5.7[2.4] \mu \mathrm{m}, p=0.017$; GCIPL: B $\left.[\mathrm{SE}]=-0.12[0.04] \mathrm{mm}^{3}, p=0.001\right)$. 
Table 3 AUC and linear regression analysis results for NMOSD vs MS, sorted in ascending order of AUC

\begin{tabular}{|c|c|c|c|c|c|c|c|c|c|}
\hline & \multirow{3}{*}{$\begin{array}{l}\text { NMOSD vs MS } \\
\text { (AUC) }\end{array}$} & \multicolumn{8}{|c|}{ Linear regression with interaction effects of diagnosis and ON history } \\
\hline & & \multicolumn{2}{|c|}{ MS vs NMOSD } & \multicolumn{2}{|l|}{ ON- vS ON+ } & \multicolumn{2}{|l|}{ NMOSD $\times$ ON } & \multirow[b]{2}{*}{$R_{\text {Marg }}^{2}$} & \multirow[b]{2}{*}{$\boldsymbol{R}_{\text {Cond }}^{2}$} \\
\hline & & B (SE) & $p$ & B (SE) & $p$ & B (SE) & $p$ & & \\
\hline Pit flat disk area $\left(\mathrm{mm}^{2}\right)$ & 0.798 & $0.011(0.005)$ & 0.021 & $0.001(0.002)$ & 0.544 & $0.015(0.004)$ & 0.001 & 0.176 & 0.843 \\
\hline $\begin{array}{l}\text { Average pit flat disk diameter } \\
(\mathrm{mm})\end{array}$ & 0.796 & $0.033(0.011)$ & 0.002 & $0.004(0.005)$ & 0.423 & $0.029(0.009)$ & 0.002 & 0.200 & 0.876 \\
\hline Minor pit flat disk length $(\mathrm{mm})$ & 0.796 & $\begin{array}{l}0.0018 \\
(0.0007)\end{array}$ & 0.011 & $\begin{array}{l}0.0002 \\
(0.0003)\end{array}$ & 0.645 & $\begin{array}{l}0.0020 \\
(0.0007)\end{array}$ & 0.007 & 0.172 & 0.827 \\
\hline Major pit flat disk length (mm) & 0.790 & $\begin{array}{l}0.0019 \\
(0.0009)\end{array}$ & 0.038 & $\begin{array}{l}0.0003 \\
(0.0004)\end{array}$ & 0.456 & $\begin{array}{l}0.0031 \\
(0.0008)\end{array}$ & $<0.001$ & 0.177 & 0.855 \\
\hline Inner rim volume $\left(\mathrm{mm}^{3}\right)$ & 0.755 & $\begin{array}{l}-0.012 \\
(0.004)\end{array}$ & 0.006 & $\begin{array}{l}-0.002 \\
(0.001)\end{array}$ & 0.170 & $\begin{array}{l}-0.007 \\
(0.003)\end{array}$ & 0.006 & 0.144 & 0.940 \\
\hline Minor slope disk length $(\mathrm{mm})$ & 0.744 & $0.017(0.006)$ & 0.013 & $0.001(0.002)$ & 0.554 & $0.008(0.004)$ & 0.051 & 0.111 & 0.941 \\
\hline Slope disk area $\left(\mathrm{mm}^{2}\right)$ & 0.739 & $0.102(0.042)$ & 0.022 & $0.010(0.011)$ & 0.371 & $0.053(0.022)$ & 0.022 & 0.096 & 0.958 \\
\hline $\begin{array}{l}\text { Average slope disk diameter } \\
(\mathrm{mm})\end{array}$ & 0.739 & $0.094(0.035)$ & 0.010 & $0.006(0.009)$ & 0.509 & $0.050(0.019)$ & 0.010 & 0.116 & 0.956 \\
\hline Major slope disk length (mm) & 0.733 & $0.017(0.008)$ & 0.040 & $0.002(0.002)$ & 0.265 & $0.010(0.004)$ & 0.019 & 0.083 & 0.963 \\
\hline Average rim height $(\mathrm{mm})$ & 0.664 & $\begin{array}{l}-0.008 \\
(0.003)\end{array}$ & 0.022 & $\begin{array}{l}-0.007 \\
(0.002)\end{array}$ & 0.001 & $\begin{array}{l}-0.009 \\
(0.004)\end{array}$ & 0.022 & 0.107 & 0.882 \\
\hline Rim volume $\left(\mathrm{mm}^{3}\right)$ & 0.627 & $\begin{array}{l}-0.053 \\
(0.032)\end{array}$ & 0.124 & $\begin{array}{l}-0.061 \\
(0.016)\end{array}$ & $<0.001$ & $\begin{array}{l}-0.044 \\
(0.032)\end{array}$ & 0.164 & 0.081 & 0.878 \\
\hline Average pit depth (mm) & 0.602 & $\begin{array}{l}-0.007 \\
(0.005)\end{array}$ & 0.147 & $\begin{array}{l}-0.007 \\
(0.002)\end{array}$ & $<0.001$ & $\begin{array}{l}-0.009 \\
(0.004)\end{array}$ & 0.025 & 0.069 & 0.931 \\
\hline Pit volume $\left(\mathrm{mm}^{3}\right)$ & 0.594 & $0.012(0.011)$ & 0.369 & $\begin{array}{l}-0.006 \\
(0.004)\end{array}$ & 0.239 & $0.002(0.008)$ & 0.845 & 0.013 & 0.917 \\
\hline $\begin{array}{l}\text { Average maximum pit slope } \\
\text { (degrees) }\end{array}$ & 0.593 & $-0.74(0.59)$ & 0.210 & $-0.73(0.26)$ & 0.008 & $-1.24(0.51)$ & 0.019 & 0.073 & 0.908 \\
\hline Major rim disk length (mm) & 0.556 & $\begin{array}{l}-0.010 \\
(0.015)\end{array}$ & 0.671 & $\begin{array}{l}-0.021 \\
(0.006)\end{array}$ & 0.002 & $\begin{array}{l}-0.005 \\
(0.013)\end{array}$ & 0.697 & 0.025 & 0.902 \\
\hline $\begin{array}{l}\text { Average rim disk diameter } \\
(\mathrm{mm})\end{array}$ & 0.550 & $\begin{array}{l}-0.014 \\
(0.026)\end{array}$ & 0.657 & $\begin{array}{l}-0.039 \\
(0.012)\end{array}$ & 0.002 & $\begin{array}{l}-0.010 \\
(0.023)\end{array}$ & 0.657 & 0.027 & 0.891 \\
\hline Rim disk area $\left(\mathrm{mm}^{2}\right)$ & 0.549 & $\begin{array}{l}-0.043 \\
(0.088)\end{array}$ & 0.628 & $\begin{array}{l}-0.128 \\
(0.039)\end{array}$ & 0.002 & $\begin{array}{l}-0.038 \\
(0.077)\end{array}$ & 0.628 & 0.027 & 0.892 \\
\hline Minor rim disk length $(\mathrm{mm})$ & 0.545 & $\begin{array}{l}-0.004 \\
(0.015)\end{array}$ & 0.761 & $\begin{array}{l}-0.022 \\
(0.007)\end{array}$ & 0.002 & $\begin{array}{l}-0.008 \\
(0.013)\end{array}$ & 0.756 & 0.029 & 0.878 \\
\hline Central foveal thickness $(\mathrm{mm})$ & 0.543 & $\begin{array}{l}-0.001 \\
(0.004)\end{array}$ & 0.891 & $\begin{array}{l}1.5 \mathrm{e}^{-4} \\
(0.001)\end{array}$ & 0.891 & $\begin{array}{l}-0.001 \\
(0.002)\end{array}$ & 0.891 & 0.002 & 0.946 \\
\hline
\end{tabular}

Abbreviations: $\mathrm{AUC}=$ area under the curve; $\mathrm{B}=$ estimate; $\mathrm{MS}=$ patients with MS; NMOSD = patients with neuromyelitis optica spectrum disorders; NMOSD $\times$ $\mathrm{ON}=$ interaction effect of diagnosis and ON; ON = optic neuritis; ON- = eyes without a history of ON; ON+ = eyes with a history of ON; $\mathrm{SE}=$ standard error of $\mathrm{B}$; $R_{\text {Cond }}^{2}=$ conditional R-squared; $R_{M a r g}^{2}=$ marginal R-squared.

Significant $p$ values and AUC $\geq 0.7$ are marked in bold.

Foveal changes may also be driven not by $\mathrm{ON}$ per se, but by the amount of neuroaxonal damage after ON. Here, MS may be a problematic control group because the amount of ONrelated retinal damage is typically lesser than in NMOSD. ${ }^{10,24}$ To investigate whether differences in neuroaxonal damage are indeed able to explain the observed foveal differences, we repeated the linear regression model analyses for the previously selected 4 parameters, but this time corrected additionally for GCIPL and INL (table 4), which can be considered sensitive parameters for ON severity. Here, it was confirmed that the observed group differences are unlikely to be explained by $\mathrm{ON}$ severity differences alone.

See supplemental data for more results on regression analysis corrected for the neuroaxonal damage (table e-3, links.lww. com/NXI/A272). Figure 2, H-I shows rim volume and average pit depth as example foveal shape parameters significantly dependent on ON status but not on diagnosis. Figure 2, 
A. NMOSD vs MS

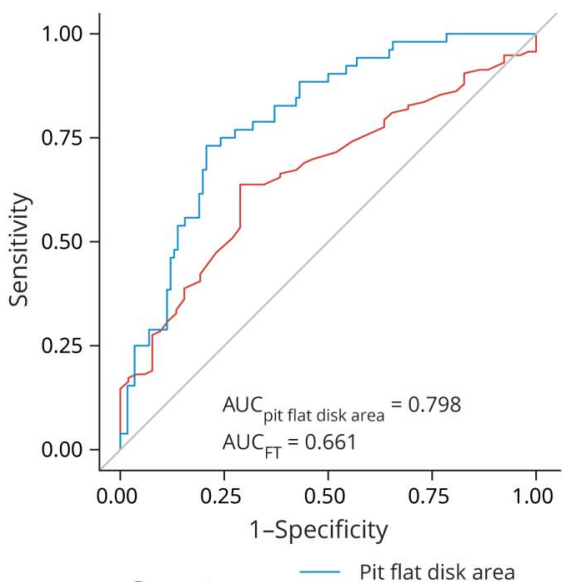

B. NMOSD ON- vs MS ON-

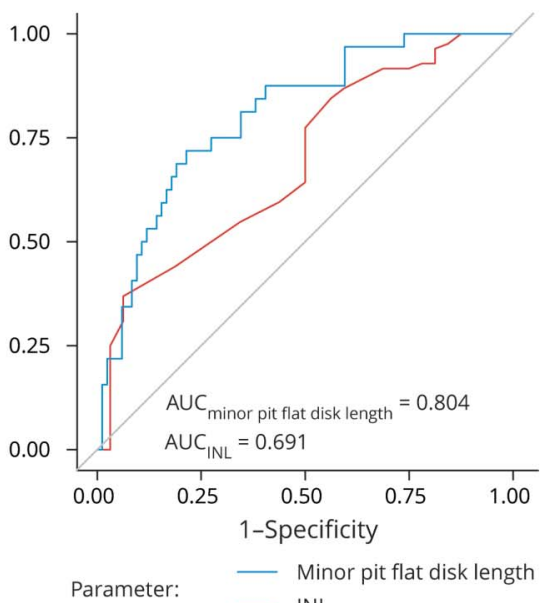

C. NMOSD ON+ vs MS ON+

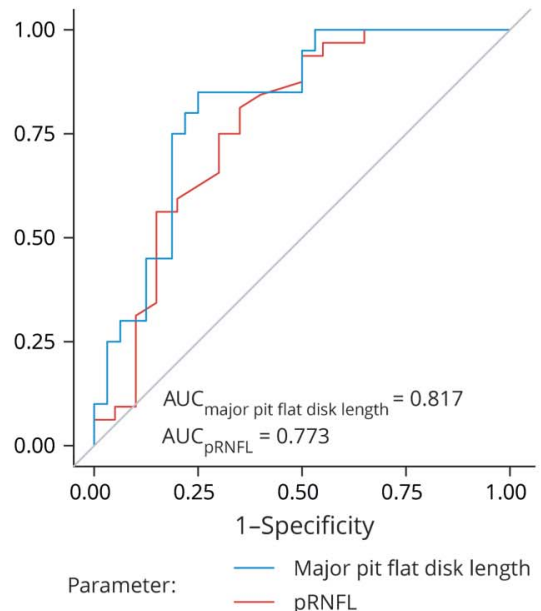

D

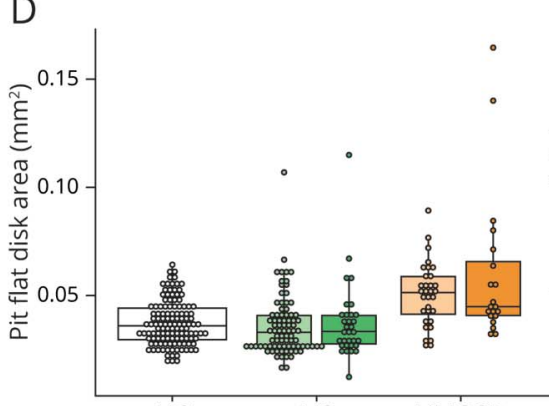

G

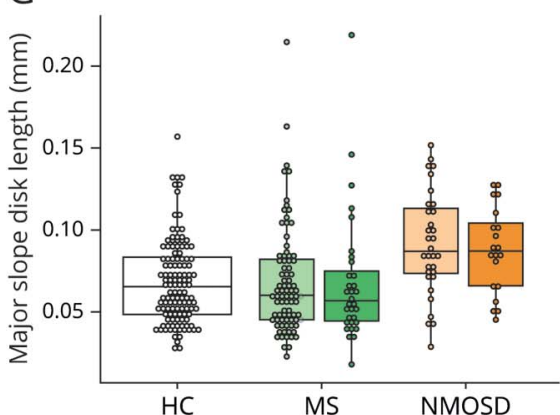

E

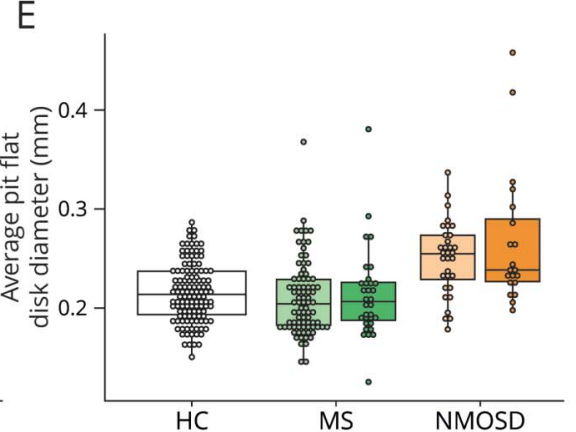

$\mathrm{H}$

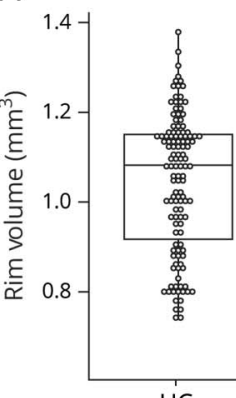

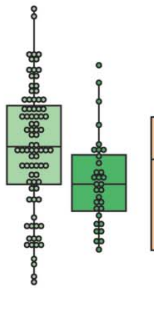

MS

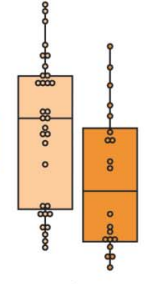

NMOSD
F

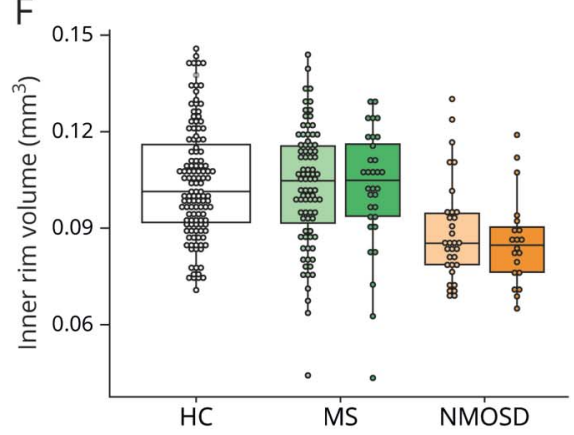

।

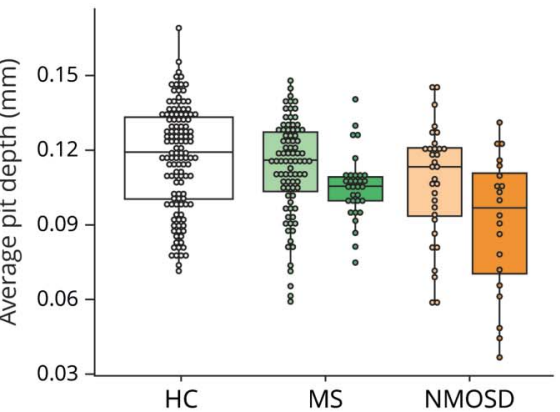

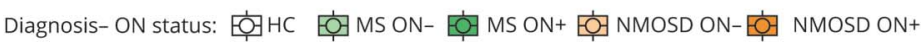
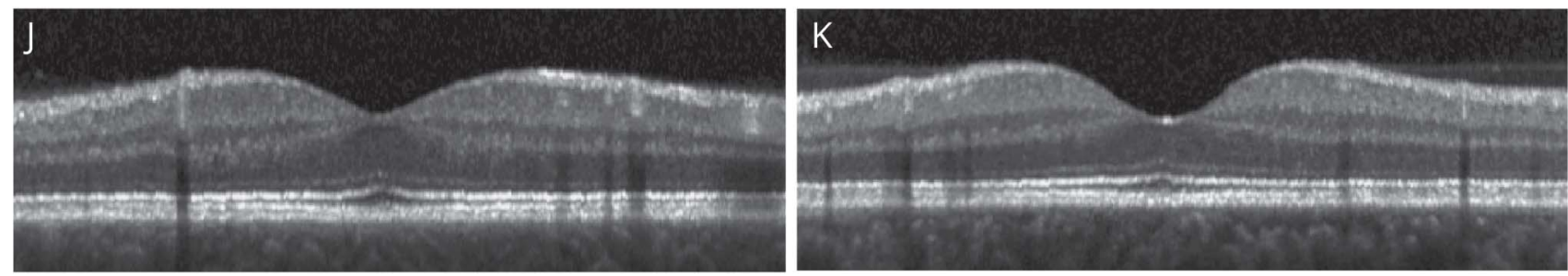

ROC curves for best-performing foveal shape and standard OCT parameters discriminating between (A) NMOSD vs MS, (B) NMOSD ON- vs MS ON-, and (C) NMOSD ON + vs MS ON+. Box and dot plots for (D) pit flat disk area, (E) average pit flat disk diameter, (F) inner rim volume, and (G) major slope disk length, the selected 4 foveal shape parameters. (H) Rim volume and (I) average pit depth, as example foveal shape parameters affected by ON but not diagnosis. A sample central (foveal) B scan of (J) MS ON- and (K) NMOSD ON-, chosen from the median of the selected pit flat disk parameters in each group, demonstrating the difference in foveal pit (pit flat disk), between NMOSD and MS in eyes without a history of ON. AUC = area under the curve; FT = foveal thickness; pRNFL = peripapillary retinal nerve fiber layer thickness; INL = inner nuclear layer volume; $\mathrm{HC}=\mathrm{healthy}$ controls; $\mathrm{MS}=$ patients with MS; NMOSD = patients with neuromyelitis optica spectrum disorders; ON = optic neuritis; ON- = eyes without a history of ON; ON+ = eyes with a history of ON; ROC = receiver operating characteristic. 
Table 4 Linear regression results for the selected foveal shape analysis parameters corrected for GCIPL and INL

\begin{tabular}{|c|c|c|c|c|c|c|c|c|c|c|}
\hline & \multicolumn{10}{|c|}{ Linear regression with interaction effects of diagnosis and ON history corrected for GCIPL } \\
\hline & \multicolumn{2}{|c|}{ MS vs NMOSD } & \multicolumn{2}{|l|}{ ON- vs ON+ } & \multicolumn{2}{|c|}{ GCIPL $\left(\mathrm{mm}^{3}\right)$} & \multicolumn{2}{|c|}{ NMOSD $\times$ ON } & \multirow[b]{2}{*}{$R_{\text {Marg }}^{2}$} & \multirow[b]{2}{*}{$\boldsymbol{R}_{\text {Cond }}^{2}$} \\
\hline & B (SE) & $p$ & B (SE) & $p$ & B (SE) & $p$ & B (SE) & $p$ & & \\
\hline Pit flat disk area $\left(\mathrm{mm}^{2}\right)$ & $\begin{array}{l}0.010 \\
(0.005)\end{array}$ & 0.031 & $\begin{array}{l}-3.2 \mathrm{e}^{-4} \\
(0.002)\end{array}$ & 0.886 & $\begin{array}{l}-0.016 \\
(0.006)\end{array}$ & 0.010 & $\begin{array}{l}0.012 \\
(0.004)\end{array}$ & 0.010 & 0.212 & 0.858 \\
\hline $\begin{array}{l}\text { Average pit flat disk diameter } \\
(\mathrm{mm})\end{array}$ & $\begin{array}{l}0.030 \\
(0.011)\end{array}$ & 0.006 & $\begin{array}{l}-8.3 e^{-5} \\
(0.005)\end{array}$ & 0.986 & $\begin{array}{l}-0.038 \\
(0.013)\end{array}$ & 0.006 & $\begin{array}{l}0.023 \\
(0.009)\end{array}$ & 0.019 & 0.230 & 0.889 \\
\hline Inner rim volume $\left(\mathrm{mm}^{3}\right)$ & $\begin{array}{l}-0.011 \\
(0.004)\end{array}$ & 0.013 & $\begin{array}{l}-7.7 e^{-4} \\
(0.001)\end{array}$ & 0.554 & $\begin{array}{l}0.010 \\
(0.004)\end{array}$ & 0.013 & $\begin{array}{l}-0.006 \\
(0.003)\end{array}$ & 0.038 & 0.159 & 0.945 \\
\hline \multirow[t]{4}{*}{ Major slope disk length $(\mathrm{mm})$} & $\begin{array}{l}0.017 \\
(0.008)\end{array}$ & 0.051 & $0.002(0.002)$ & 0.360 & $\begin{array}{l}5.5 e^{-5} \\
(0.006)\end{array}$ & 0.992 & $\begin{array}{l}0.010 \\
(0.004)\end{array}$ & 0.033 & 0.082 & 0.962 \\
\hline & \multicolumn{10}{|c|}{ Linear regression with interaction effects of diagnosis and ON history corrected for INL } \\
\hline & \multicolumn{2}{|c|}{ MS vs NMOSD } & \multicolumn{2}{|l|}{ ON- vs ON+ } & \multicolumn{2}{|c|}{ INL $\left(\mathrm{mm}^{3}\right)$} & \multicolumn{2}{|c|}{ NMOSD $\times$ ON } & & \\
\hline & B (SE) & $p$ & B (SE) & $p$ & B (SE) & $p$ & B (SE) & $p$ & $R_{\text {Marg }}^{2}$ & $R_{\text {Cond }}^{2}$ \\
\hline Pit flat disk area $\left(\mathrm{mm}^{2}\right)$ & $\begin{array}{l}0.012 \\
(0.005)\end{array}$ & 0.017 & $\begin{array}{l}0.001 \\
(0.002)\end{array}$ & 0.771 & $\begin{array}{l}0.041 \\
(0.024)\end{array}$ & 0.145 & $\begin{array}{l}0.013 \\
(0.004)\end{array}$ & 0.014 & 0.193 & 0.851 \\
\hline $\begin{array}{l}\text { Average pit flat disk diameter } \\
(\mathrm{mm})\end{array}$ & $\begin{array}{l}0.035 \\
(0.011)\end{array}$ & 0.005 & $\begin{array}{l}0.003 \\
(0.005)\end{array}$ & 0.482 & $\begin{array}{l}0.075 \\
(0.054)\end{array}$ & 0.200 & $\begin{array}{l}0.027 \\
(0.010)\end{array}$ & 0.013 & 0.208 & 0.882 \\
\hline Inner rim volume $\left(\mathrm{mm}^{3}\right)$ & $\begin{array}{l}-0.012 \\
(0.004)\end{array}$ & 0.009 & $\begin{array}{l}-0.002 \\
(0.001)\end{array}$ & 0.212 & $\begin{array}{l}0.003 \\
(0.018)\end{array}$ & 0.860 & $\begin{array}{l}-0.008 \\
(0.003)\end{array}$ & 0.009 & 0.143 & 0.939 \\
\hline Major slope disk length (mm) & $\begin{array}{l}0.016 \\
(0.008)\end{array}$ & 0.061 & $\begin{array}{l}0.002 \\
(0.002)\end{array}$ & 0.312 & $\begin{array}{l}-0.014 \\
(0.028)\end{array}$ & 0.623 & $\begin{array}{l}0.010 \\
(0.004)\end{array}$ & 0.022 & 0.083 & 0.962 \\
\hline
\end{tabular}

Abbreviations: $\mathrm{B}$ = estimate; $\mathrm{GCIPL}=$ combined macular ganglion cell and inner plexiform layer volume; INL = inner nuclear layer volume; $\mathrm{MS}=$ patients with MS; NMOSD = patients with neuromyelitis optica spectrum disorders; NMOSD $\times$ ON = interaction effect of diagnosis and ON; ON = optic neuritis; ON- = eyes without a history of ON; ON+ = eyes with a history of ON; $\mathrm{SE}=$ standard error of $\mathrm{B} ; R_{\text {Cond }}^{2}=$ conditional R-squared; $R_{\text {Marg }}^{2}=$ marginal R-squared.

Significant $p$ values are marked in bold.

J-K shows sample central B scans (crossing the fovea) of $\mathrm{ON}$ - from patients with NMOSD and MS, chosen from the median of the selected pit flat disk parameters in each group.

\section{Parameter confirmation}

Finally, we tested whether the parameters identified in the exploratory analysis could be confirmed in an independent cohort of patients with NMOSD and MS measured with the same device and protocol at an independent center. Based on differences in the selected parameters, we determined the minimum sample size for a confirmatory cohort as $\mathrm{n}=38,29,35$, and 59 eyes per group, based on measurements for pit flat disk area, average pit flat disk diameter, inner rim volume, and major slope disk length, respectively. In this confirmatory cohort, pit flat disk area and average pit flat disk diameter were confirmed to be significantly different in NMOSD in comparison to MS (B $[\mathrm{SE}]=0.007[0.004] \mathrm{mm}^{2}, p=0.035$ and $\mathrm{B}[\mathrm{SE}]=0.018$ $[0.010] \mathrm{mm}, p=0.039$, respectively), neither dependent on ON $(p=0.254$ and 0.184$)$ nor on NMOSD-specific ON $(p=0.293$ and 0.382 ). Differences in inner rim volume were not significant in the confirmatory cohort (diagnosis: $p=0.125$; ON: $p=$ 0.080; NMOSD-specific ON: $p=0.056$ ). Major slope disk length only showed a significant association with NMOSDspecific ON (diagnosis: $p=0.155$; ON history: $p=0.370$; NMOSD-specific ON: B $[\mathrm{SE}]=0.012[0.007] \mathrm{mm}, p=0.046)$.

\section{Discussion}

Using a novel foveal morphometry approach, we here show that foveal shape is altered in patients with AQP4IgG-seropositive NMOSD. Our results further support that these changes cannot be explained by neuroaxonal damage resulting from $\mathrm{ON}$ alone.

Foveal morphometry described a flatter and wider fovea in AQP4-IgG-seropositive NMOSD both in comparison to MS and $\mathrm{HC}$ (figure 2, J-K). This is characterized by increased pit flat disk area, increased average pit flat disk diameter, reduced inner rim volume, and increased major slope disk length. Although neuroaxonal damage from $\mathrm{ON}$ altered the foveal shape as well, we observed robust changes in these parameters also in eyes never experiencing an $\mathrm{ON}$ and when correcting for $\mathrm{ON}$ or neuroaxonal damage in the statistical models in all eyes.

The foveal shape changes in AQP4-IgG-seropositive NMOSD reported in our study are supported by previous studies, which investigated thickness or volume changes as indirect evidence for foveal shape changes. Jeong et al. ${ }^{16}$ and Oertel et al. ${ }^{17}$ showed a significant reduction in FT in eyes of patients with AQP4-IgG-seropositive NMOSD independent of $\mathrm{ON}$ in comparison to HCs. 
A pathophysiologic explanation for the observed changes could be the presence of a primary retinopathy in AQP4-IgG-seropositive NMOSD, mediated by AQP4-IgG. The principal glial cell of the retina is the Müller cell, expresses AQP4, and is enriched around the fovea. ${ }^{25}$ Müller cell bodies reside in the INL, but process stretch through the whole thickness of the retina, linking retinal neurons and photoreceptors with blood vessels. Importantly, animal studies have shown complement-independent AQP4 loss in Müller cells in rats induced by AQP4-IgG, which is in line with an AQP4-IgG-mediated primary retinopathy in NMOSD. ${ }^{26,27}$ AQP4-IgG-mediated primary retinal astrocytopathy has been also suggested in human by AQP4-IgG-seropositive NMOSD autopsy cases. ${ }^{28}$ AQP4 is expressed in Müller cell end feetanalogous to astrocytic end feet-at the blood-retina barrier. ${ }^{29}$ For AQP4-IgG circulating in serum to reach its antigen, the bloodretina barrier presumably needs to be disrupted. Blood-retina or -brain barrier disruptions are typically associated with an acute inflammatory event and then conceptionally linked to an acute attack involving complement. ${ }^{30}$ It is unclear whether or to which extent blood-retina/brain barrier disruptions occur in NMOSD and other diseases that do not lead to full attack cascades. A recent analysis of the NMOSD momentum trial data ${ }^{31}$ revealed that elevated glial fibrillary acidic protein levels in serum were associated with an increased attack risk, independently suggesting that there is indeed subclinical astrocyte damage outside attacks (Annual European Committee for Treatment and Research in Multiple Sclerosis [ECTRIMS] 2019, P1609). ${ }^{32}$ A recent study could further show that in rats, blood-brain barrier breakdown is not necessary for NMOSD pathology, but that NMOSD-like disease can be caused by AQP4-IgG circulating in CSF. ${ }^{33}$ We recently reported progressive GCIPL loss without $\mathrm{ON}$ in a longitudinal study investigating an overlapping cohort. ${ }^{34}$ Further evidence for a primary retinopathy comes from Tian et al. ${ }^{35}$ reporting inner retinal layer thinning independent from ON. Significant changes in vascularization of the fovea were also shown in patients with AQP4-IgG-seropositive NMOSD in comparison to HCs using OCT angiography. ${ }^{36-38}$

Alternatively, foveal changes could be caused by subclinical ON. Occasionally, studies have reported neuroaxonal damage also in eyes without prior ON in AQP4-IgG-seropositive NMOSD, which could be interpreted as evidence as such. ${ }^{39}$ However, earlier studies had cohort heterogeneity due to incomplete antibody characterization or inclusion of patients with antibody-negative NMOSD. Furthermore, ON in NMOSD often occurs near the chiasm, and neuroaxonal damage can be caused by chiasmal crossover from an affected eye to the fellow eye, ${ }^{40}$ which might not be clinically apparent. In this study, we found evidence of neuroaxonal damage also in eyes reported to have never experienced $\mathrm{ON}$, which is in agreement with our recent findings in a multicenter study. ${ }^{34}$ It is possible that our data set also included fellow eyes that were affected from cross-chiasmal affects during a contralateral $\mathrm{ON}$. The number of patients with NMOSD only experiencing transverse myelitis and no $\mathrm{ON}$ was too small, which is why we refrained from analyzing eyes of these patients separately. In consequence, we cannot fully determine to which extent the observed changes may be caused or affected by covert ON and neuroaxonal damage of the optic nerve.

The main limitation of our study was the low sample size for AQP4 IgG-seropositive NMOSD, especially for patients without a history of $\mathrm{ON}$, which is unfortunately common in studies investigating NMOSD. Another limitation of this study is that the diagnostic value is unclear, as we only used scans from 1 OCT device using a single scanning protocol. It is unclear how scans from different OCT devices and scanning protocols can be compared.

Foveal morphometry may potentially be useful for differential diagnosis of AQP4-IgG-seropositive NMOSD. Typically, pRNFL and GCIPL as well as other OCT parameters associated with neuroaxonal damage are mostly nonspecific to the underlying ON etiology. In contrast, many foveal morphometry parameters showed significant differences between patients with NMOSD and MS in this study. Parameter selection resulted in 4 promising parameters describing foveal differences: pit flat disk area, average pit flat disk diameter, major slope disk length, and inner rim volume. Only the first 2 parameters could be confirmed in an independent cohort. The reason may be the different frequency of $\mathrm{ON}$ in the confirmatory cohort between patients with MS and NMOSD, which exemplifies the need for additional confirmation, especially in eyes that are inconspicuous in regard to neuroaxonal damage from ON. Future work should further investigate this by comparing patients with myelin oligodendrocyte glycoprotein (MOG)-IgG-seropositive disease against patients with MOG/ AQP4-IgG double-negative NMOSD, as well as clarify effects of scan protocols and foveal variability in healthy persons.

\section{Study funding}

Supported by the Einstein Foundation Berlin (Einstein Junior Scholarship to S.M.), the German Federal Ministry of Economic Affairs and Energy (BMWI EXIST 03EFEBE079 to A.U.B. and E.M.K.), German Research Foundation (DFG Exc. 257 to F.P. and A.U.B.), German Federal Ministry of Education and Research (BMBF Neu ${ }^{2}$ ADVISIMS to F.P. and A.U.B. as well as part of the "German Competence Network Multiple Sclerosis" (KKNMS), project NationNMO, 01GI1602B to O.A.), and Novartis (research grant to H.G.Z.).

\section{Disclosure}

E.M. Kadas, S.K. Yadav, A.U. Brandt, S. Motamedi, and F. Paul are named as coinventors on the patent application for the foveal shape analysis method used by this manuscript ("Method for estimating shape parameters of the fovea by optical coherence tomography", International Publication Number: "WO 2019/016319 A1"). E.M. Kadas, S.K. Yadav, F. Paul, and A.U. Brandt are cofounders and hold shares in technology start-up Nocturne GmbH, which has commercial interest in OCT applications in neurology. E.M. Kadas and S.K. Yadav are now employees of Nocturne GmbH. H.G. Zimmermann received a research grant from Novartis. All other authors report no relevant disclosures. Go to Neurology.org/NN for full disclosures. 


\section{Publication history}

Received by Neurology: Neuroimmunology \& Neuroinflammation January 30, 2020. Accepted in final form April 16, 2020.

\section{Appendix Authors}

\begin{tabular}{lll}
\hline Name & Location & Contribution \\
$\begin{array}{l}\text { Seyedamirhosein } \\
\text { Motamedi, MSc }\end{array}$ & $\begin{array}{l}\text { Charité-Universitätsmedizin } \\
\text { Berlin, Germany }\end{array}$ & $\begin{array}{l}\text { Collected the data; conducted } \\
\text { the statistical analysis; } \\
\text { contributed to development of } \\
\text { the foveal shape analysis } \\
\text { method; and drafted the } \\
\text { manuscript for intellectual } \\
\text { content }\end{array}$ \\
& & \\
\hline $\begin{array}{l}\text { Frederike C. } \\
\text { Oertel, MD }\end{array}$ & Charité-Universitätsmedizin & $\begin{array}{l}\text { Collected the data; performed } \\
\text { OCT quality control and } \\
\text { segmentation; contributed to } \\
\text { data interpretation; and } \\
\text { revised the manuscript for } \\
\text { intellectual content }\end{array}$ \\
& &
\end{tabular}

\begin{tabular}{lll}
\hline $\begin{array}{l}\text { Sunil K. Yadav, } \\
\text { PhD }\end{array}$ & $\begin{array}{l}\text { Charité-Universitätsmedizin } \\
\text { Berlin, Germany }\end{array}$ & $\begin{array}{l}\text { Developed the foveal shape } \\
\text { analysis method and revised } \\
\text { the manuscript for } \\
\text { intellectual content }\end{array}$ \\
\hline Ella M. Kadas, PhD & $\begin{array}{l}\text { Charité-Universitätsmedizin } \\
\text { Berlin, Germany }\end{array}$ & $\begin{array}{l}\text { Contributed to development } \\
\text { of the foveal shape analysis } \\
\text { method and revised the } \\
\text { manuscript for intellectual } \\
\text { content }\end{array}$
\end{tabular}

\begin{tabular}{lll}
\hline Margit Weise, & Heinrich Heine University, & $\begin{array}{l}\text { Major role in acquisition of } \\
\text { the confirmatory data and } \\
\text { MSc }\end{array}$ \\
& Düsseldorf, Germany & intellectual content
\end{tabular}

\begin{tabular}{lll}
\hline Joachim Havla, & Ludwig-Maximilians & $\begin{array}{l}\text { Contributed to data } \\
\text { MD }\end{array}$ \\
& University, Munich, Germany & $\begin{array}{l}\text { interpretation and revised the } \\
\text { manuscript for intellectual } \\
\text { content }\end{array}$
\end{tabular}

\begin{tabular}{lll}
\hline Marius & Heinrich Heine University, & $\begin{array}{l}\text { Major role in acquisition of } \\
\text { the confirmatory data and } \\
\text { revised the manuscript for } \\
\text { intellectual content }\end{array}$ \\
\hline
\end{tabular}

\begin{tabular}{lll}
\hline Orhan Aktas, MD & $\begin{array}{l}\text { Heinrich Heine University, } \\
\text { Düsseldorf, Germany }\end{array}$ & $\begin{array}{l}\text { Major role in acquisition of } \\
\text { the confirmatory data and } \\
\text { revised the manuscript for } \\
\text { intellectual content }\end{array}$
\end{tabular}

\begin{tabular}{lll}
\hline Philipp Albrecht, & Heinrich Heine University, & $\begin{array}{l}\text { Major role in acquisition of } \\
\text { the confirmatory data and } \\
\text { MD }\end{array}$ \\
& Düsseldorf, Germany the manuscript for \\
& intellectual content
\end{tabular}

\begin{tabular}{lll}
\hline Klemens & Charité-Universitätsmedizin & Contributed to study \\
Ruprecht, MD & Berlin, Germany & $\begin{array}{l}\text { management and revised the } \\
\text { manuscript for intellectual }\end{array}$
\end{tabular}
content

\begin{tabular}{lll}
\hline $\begin{array}{l}\text { Judith Bellmann- } \\
\text { Strobl, MD }\end{array}$ & $\begin{array}{l}\text { Charité-Universitätsmedizin } \\
\text { Berlin, Germany }\end{array}$ & $\begin{array}{l}\text { Contributed to study } \\
\text { management and revised the } \\
\text { manuscript for intellectual } \\
\text { content }\end{array}$ \\
\hline $\begin{array}{l}\text { Hanna G. } \\
\text { PhD }\end{array}$ & $\begin{array}{l}\text { Charité-Universitätsmedizin } \\
\text { Berlin, Germany }\end{array}$ & $\begin{array}{l}\text { Contributed to data } \\
\text { interpretation and revised the } \\
\text { manuscript for intellectual } \\
\text { content }\end{array}$ \\
\hline $\begin{array}{l}\text { Friedemann Paul, } \\
\text { MD }\end{array}$ & Charité-Universitätsmedizin & $\begin{array}{l}\text { Contributed to study } \\
\text { management; contributed to } \\
\text { data interpretation; and } \\
\text { revised the manuscript for } \\
\text { intellectual content }\end{array}$ \\
& Berlin, Germany & $\begin{array}{l}\text { Designed and conceptualized } \\
\text { the study; supervised the } \\
\text { statistical analysis; and } \\
\text { drafted the manuscript for } \\
\text { intellectual content }\end{array}$ \\
\hline $\begin{array}{l}\text { Alexander U. } \\
\text { Brandt, MD }\end{array}$ & Charité-Universitätsmedizin & Berlin, Germany \\
& &
\end{tabular}

\section{References}

1. Jarius S, Paul F, Franciotta D, et al. Mechanisms of disease: aquaporin-4 antibodies in neuromyelitis optica. Nat Clin Pract Neurol 2008;4:202-214.

2. Paul F, Jarius S, Aktas O, et al. Antibody to aquaporin 4 in the diagnosis of neuromyelitis optica. PLoS Med 2007;4:e133.

3. Zekeridou A, Lennon VA. Aquaporin-4 autoimmunity. Neurol Neuroimmunol Neuroinflamm 2015;2:e110. doi: 10.1212/NXI.0000000000000110.

4. Jarius S, Wildemann B, Paul F. Neuromyelitis optica: clinical features, immunopathogenesis and treatment: neuromyelitis optica. Clin Exp Immunol 2014;176:149-164.

5. Schmidt F, Zimmermann H, Mikolajczak J, et al. Severe structural and functional visual system damage leads to profound loss of vision-related quality of life in patients with neuromyelitis optica spectrum disorders. Mult Scler Relat Disord 2017;11:45-50.

6. Schneider E, Zimmermann H, Oberwahrenbrock T, et al. Optical coherence tomography reveals distinct patterns of retinal damage in neuromyelitis optica and multiple sclerosis. PLoS One 2013;8:e66151.

7. Wingerchuk DM, Banwell B, Bennett JL, et al. International consensus diagnostic criteria for neuromyelitis optica spectrum disorders. Neurology 2015;85:177-189.

8. Yamamura T, Nakashima I. Foveal thinning in neuromyelitis optica: a sign of retinal astrocytopathy? Neurol Neuroimmunol Neuroinflamm 2017;4:e347. doi: 10.1212/ NXI.0000000000000347.

9. Oertel FC, Zimmermann H, Paul F, Brandt AU. Optical coherence tomography in neuromyelitis optica spectrum disorders: potential advantages for individualized monitoring of progression and therapy. EPMA J 2018;9:21-33.

10. Bennett JL, de Seze J, Lana-Peixoto $M$, et al. Neuromyelitis optica and multiple sclerosis: seeing differences through optical coherence tomography. Mult Scler Houndmills Basingstoke Engl 2015;21:678-688.

11. Oertel FC, Zimmermann H, Mikolajczak J, et al. Contribution of blood vessels to retinal nerve fiber layer thickness in NMOSD. Neurol Neuroimmunol Neuroinflamm 2017;4:e338. doi: 10.1212/NXI.0000000000000338.

12. Oberwahrenbrock T, Traber GL, Lukas S, et al. Multicenter reliability of semiautomatic retinal layer segmentation using OCT. Neurol Neuroimmunol Neuroinflamm 2018;5:e449. doi: 10.1212/NXI.0000000000000449.

13. Kaufhold F, Zimmermann H, Schneider E, et al. Optic neuritis is associated with inner nuclear layer thickening and microcystic macular edema independently of multiple sclerosis. PLoS One 2013;8:e71145.

14. Syc SB, Saidha S, Newsome SD, et al. Optical coherence tomography segmentation reveals ganglion cell layer pathology after optic neuritis. Brain 2012;135:521-533.

15. Pache F, Zimmermann H, Mikolajczak J, et al. MOG-IgG in NMO and related disorders: a multicenter study of 50 patients. Part 4: afferent visual system damage after optic neuritis in MOG-IgG-seropositive versus AQP4-IgG-seropositive patients. J Neuroinflammation 2016;13:282.

16. Jeong IH, Kim HJ, Kim NH, Jeong KS, Park CY. Subclinical primary retinal pathology in neuromyelitis optica spectrum disorder. J Neurol 2016;263:1343-1348.

17. Oertel FC, Kuchling J, Zimmermann H, et al. Microstructural visual system changes in AQP4-antibody-seropositive NMOSD. Neurol Neuroimmunol Neuroinflamm 2017; 4:e334. doi: 10.1212/NXI.0000000000000334.

18. Yadav SK, Motamedi S, Oberwahrenbrock T, et al. CuBe: parametric modeling of $3 \mathrm{D}$ foveal shape using cubic Bézier. Biomed Opt Express 2017;8:4181.

19. Tewarie P, Balk L, Costello $\mathrm{F}$, et al. The OSCAR-IB consensus criteria for retinal OCT quality assessment. PLoS One 2012;7:e34823.

20. Polman CH, Reingold SC, Banwell B, et al. Diagnostic criteria for multiple sclerosis: 2010 revisions to the McDonald criteria. Ann Neurol 2011;69:292-302.

21. Schippling S, Balk LJ, Costello F, et al. Quality control for retinal OCT in multiple sclerosis: validation of the OSCAR-IB criteria. Mult Scler Houndmills Basingstoke Engl 2015;21:163-170.

22. Cruz-Herranz A, Balk LJ, Oberwahrenbrock T, et al. The APOSTEL recommendations for reporting quantitative optical coherence tomography studies. Neurology 2016;86:2303-2309.

23. R Core Team. R: A Language and Environment for Statistical Computing [online]. Vienna: R Foundation for Statistical Computing; 2018. Available at: www.R-project.org/. Accessed June 13, 2019.

24. Ratchford JN, Quigg ME, Conger A, et al. Optical coherence tomography helps differentiate neuromyelitis optica and MS optic neuropathies. Neurology 2009;73:302-308.

25. Bringmann A, Pannicke T, Grosche J, et al. Müller cells in the healthy and diseased retina. Prog Retin Eye Res 2006;25:397-424.

26. Felix CM, Levin MH, Verkman AS. Complement-independent retinal pathology produced by intravitreal injection of neuromyelitis optica immunoglobulin G. J Neuroinflammation 2016;13:275

27. Zeka B, Hastermann M, Kaufmann N, et al. Aquaporin 4-specific T cells and NMOIgG cause primary retinal damage in experimental NMO/SD. Acta Neuropathol Commun 2016;4:82

28. Hokari M, Yokoseki A, Arakawa M, et al. Clinicopathological features in anterior visual pathway in neuromyelitis optica. Ann Neurol 2016;79:605-624.

29. Goodyear MJ, Crewther SG, Junghans BM. A role for aquaporin-4 in fluid regulation in the inner retina. Vis Neurosci 2009;26:159-165.

30. Takeshita Y, Obermeier B, Cotleur AC, et al. Effects of neuromyelitis optica-IgG at the blood-brain barrier in vitro. Neurol Neuroimmunol Neuroinflamm 2017;4:e311. doi: 10.1212/NXI.0000000000000311.

31. Cree BAC, Bennett JL, Kim HJ, et al. Inebilizumab for the treatment of neuromyelitis optica spectrum disorder (N-MOmentum): a double-blind, randomised placebocontrolled phase 2/3 trial. Lancet Lond Engl 2019;394:1352-1363.

32. ECTRIMS 2019-late breaking news abstracts. Mult Scler J 2019;25:890-938 
33. Hillebrand S, Schanda K, Nigritinou M, et al. Circulating AQP4-specific autoantibodies alone can induce neuromyelitis optica spectrum disorder in the rat. Acta Neuropathol (Berl) 2019;137:467-485.

34. Oertel FC, Havla J, Roca-Fernández A, et al. Retinal ganglion cell loss in neuromyelitis optica: a longitudinal study. J Neurol Neurosurg Psychiatry 2018;89: 1259-1265.

35. Tian DC, Su L, Fan M, et al. Bidirectional degeneration in the visual pathway in neuromyelitis optica spectrum disorder (NMOSD). Mult Scler J 2018;24: 1585-1593.

36. Huang Y, Zhou L, ZhangBao J, et al. Peripapillary and parafoveal vascular network assessment by optical coherence tomography angiography in aquaporin-4 antibodypositive neuromyelitis optica spectrum disorders. Br J Ophthalmol 2019;103: 789-796.
37. Kwapong WR, Peng C, He Z, Zhuang X, Shen M, Lu F. Altered macular microvasculature in neuromyelitis optica spectrum disorders. Am J Ophthalmol 2018;192: 47-55.

38. Green AJ, Cree BAC. Distinctive retinal nerve fibre layer and vascular changes in neuromyelitis optica following optic neuritis. J Neurol Neurosurg Psychiatry 2009;80: 1002-1005.

39. Ringelstein M, Harmel J, Zimmermann H, et al. Longitudinal optic neuritis-unrelated visual evoked potential changes in NMO spectrum disorders. Neurology 2020;94: e407-e418.

40. Ramanathan S, Prelog K, Barnes EH, et al. Radiological differentiation of optic neuritis with myelin oligodendrocyte glycoprotein antibodies, aquaporin-4 antibodies, and multiple sclerosis. Mult Scler Houndmills Basingstoke Engl 2016;22:470-482. 


\section{Neurology \\ Neuroimmunology \& Neuroinflammation}

\section{Altered fovea in AQP4-IgG-seropositive neuromyelitis optica spectrum disorders}

Seyedamirhosein Motamedi, Frederike C. Oertel, Sunil K. Yadav, et al.

Neurol Neuroimmunol Neuroinflamm 2020;7;

DOI 10.1212/NXI.0000000000000805

This information is current as of June 23, 2020

\section{Updated Information \& Services}

References

Citations

Subspecialty Collections

Permissions \& Licensing

Reprints including high resolution figures, can be found at:

http://nn.neurology.org/content/7/5/e805.full.html

This article cites 39 articles, 9 of which you can access for free at: http://nn.neurology.org/content/7/5/e805.full.html\#\#ref-list-1

This article has been cited by 3 HighWire-hosted articles: http://nn.neurology.org/content/7/5/e805.full.html\#\#otherarticles

This article, along with others on similar topics, appears in the following collection(s):

Devic's syndrome

http://nn.neurology.org//cgi/collection/devics_syndrome

Retina

http://nn.neurology.org//cgi/collection/retina

Information about reproducing this article in parts (figures,tables) or in its entirety can be found online at:

http://nn.neurology.org/misc/about.xhtml\#permissions

Information about ordering reprints can be found online:

http://nn.neurology.org/misc/addir.xhtml\#reprintsus

Neurol Neuroimmunol Neuroinflamm is an official journal of the American Academy of Neurology.

Published since April 2014, it is an open-access, online-only, continuous publication journal. Copyright

Copyright $\left({ }^{\circ} 2020\right.$ The Author(s). Published by Wolters Kluwer Health, Inc. on behalf of the American

Academy of Neurology.. All rights reserved. Online ISSN: 2332-7812.

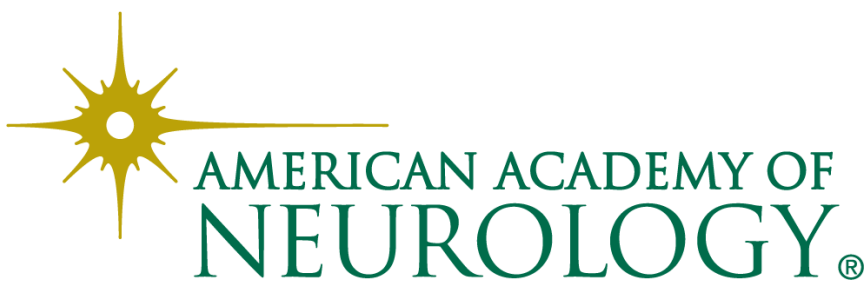

